Check for updates

Cite this: Nanoscale Adv., 2019, 1, 1175

\section{Spontaneous directional motion of water molecules in single-walled carbon nanotubes with a stiffness gradient $\uparrow$}

\begin{abstract}
Shuai Chen, (D) Yuan Cheng, (D) Gang Zhang (D)* and Yong-Wei Zhang
Controlling water molecular motion at the nanoscale is critical for many important applications, such as water splitting to produce hydrogen and oxygen, biological and chemical cell reactions, nanofluidics, drug delivery, water treatment, etc. In this paper, we propose a new nanoscale device based on carbon nanotubes (CNTs) with a stiffness gradient to create a spontaneous directional motion of water molecules, and perform molecular dynamics simulations to analyze its transport characteristics. We find that the $(6,6)$ CNT possesses an optimal water transport rate. In the thinner CNTs, the water molecules are strongly confined by the CNT wall, resulting in a higher friction force; while in the thicker CNTs, the driving force is lower, and the water molecules tend to form ring-like configurations, resulting in a slower motion. For the $(6,6)$ CNT, water molecules tend to favor a chain-like configuration, through which the molecules are able to move synergistically along the stiffness gradient, and the transportation efficiency increases with the stiffness gradient but decreases with temperature. Both energetic and kinetic analyses are performed to elucidate this fascinating directional motion. Our work demonstrates a new strategy for controlling water molecular motion at the nanoscale without resorting to any active driving source, such as electric field, temperature or pressure difference.
\end{abstract}

Received 1st November 2018 Accepted 19th December 2018

DOI: $10.1039 / \mathrm{c} 8 \mathrm{na00322 \textrm {j }}$

rsc.li/nanoscale-advances of nanotubes ${ }^{16}$ and dopant atoms ${ }^{17}$ may greatly affect the water permeation rate. In multi-walled carbon nanotubes (MWCNTs), the nanotube type and layer number may also exert strong influence on the water transport behaviors. ${ }^{\mathbf{1 8 , 1 9}}$ These studies on water molecules inside CNTs have not only unveiled new physics for confined water molecules, but also encouraged researchers to explore novel strategies to control the transport behaviors of water molecules at the nanoscale. Furthermore, the recent advances in precise and controllable fabrication of $\mathrm{CNTs}^{\mathbf{2 8 , 2 9}}$ also pave the way towards efficient water transport at the nanoscale.

The directional motion of water molecules in CNTs has been typically realized by active means, including applications of pressure, ${ }^{16,25}$ electrical currents ${ }^{30}$ (or charge ${ }^{12,31}$ ), selective heating $^{\mathbf{1 3 , 1 5}}$ or complicated chemical reactions. ${ }^{32}$ For instance, Ma et $a .^{25}$ found that the water flow velocity increases linearly with pressure between the two ends of a CNT. Zuo et al. ${ }^{14}$ introduced different types of external point charges outside a CNT, and realized directional motion of water molecules through the CNT channel under the influence of both thermal fluctuation and charge affinity. The directional migration of water molecules in CNTs can also be realized by using an electrical current. ${ }^{30}$ Very recently, Oyarzua et al. ${ }^{15}$ proposed a water pump based on CNTs with an imposed constant thermal gradient. The underlying mechanism for the fluid flow is the thermally induced asymmetric oscillations along the CNT, which propel the water in a constant, whip-like motion. 
It is noted that all these aforementioned methods require active external means to drive water molecules. ${ }^{33}$ Then an interesting question is: is it possible to create a spontaneous directional motion of water molecules in CNTs without resorting to any external driving means? For this purpose, we propose a new device based on CNTs with a stiffness gradient to realize the spontaneous directional motion of water molecules. Our molecular dynamics simulations clearly show the spontaneous water motion through CNTs, without resorting to any active driving force, such as electric field, pressure or temperature difference. We further analyze the mechanism that underlies the spontaneous directional motion and factors that influence the transport rate.

\section{Simulation method}

The large-scale atomic/molecular massively parallel simulator (LAMMPS) package ${ }^{34}$ was used to carry out the simulations. The simulated system is shown in Fig. 1a. In the system, water molecules were placed at the center of an uncapped $(6,6)$ nanotube (101.84 $\AA$ long and $8.02 \AA$ in diameter). The adaptive intermolecular reactive empirical bond order (AIREBO) potential $^{35}$ was used to describe the carbon-carbon interactions within the nanotube. The water model in this study is a transferable intermolecular potential with 3-points (TIP/3P), ${ }^{36}$ which has been widely used to simulate water in molecule dynamics simulations. This potential consists of a Coulomb interaction between partial point charges on the oxygen $(-0.830 e)$ and hydrogen $(+0.415 e)$ atoms and a Lennard-Jones interaction ${ }^{37}$ with $\sigma_{\mathrm{OO}}=3.188 \AA$, and $\varepsilon_{\mathrm{OO}}=0.102 \mathrm{kcal} \mathrm{mol}^{-1}$. The particleparticle particle-mesh (PPPM) method was applied to compute the long-range Coulomb interaction. An oxygen-hydrogen bond length of $0.9572 \AA$ and a bond angle of $104.52^{\circ}$ are maintained by harmonic potentials with spring constants of $450 \mathrm{kcal}\left(\mathrm{mol}^{-1}\right.$ $\left.\AA^{2}\right)^{-1}$ and $55 \mathrm{kcal}\left(\mathrm{mol}^{-1} \operatorname{rad}^{2}\right)^{-1}$. The Lennard-Jones potential was also used to simulate the interaction between the nanotube and water with $\sigma_{\mathrm{CO}}=3.298 \AA$, and $\varepsilon_{\mathrm{CO}}=0.0828 \mathrm{kcal} \mathrm{mol}^{-1}$.

Previous studies have shown that deposition of atoms or molecules on the external surface of a CNT, either physically or chemically, may change the local potential field of carbon atoms in the CNT, causing a modulation in both the structure and physical properties of the CNT. ${ }^{38-40}$ For example, asymmetric axial thermal conductance was observed in a CNT with inhomogeneously deposited mass, as a direct demonstration of

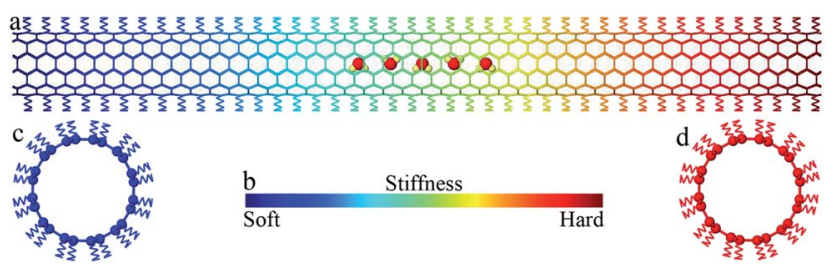

Fig. 1 (a) Initial structure of the ensemble with five water molecules in an uncapped nanotube. The colors represent the stiffness of springs linked with carbon atoms. (b) The color scale of the spring stiffness from the soft region to the hard region. Carbon atoms are linked to an underlying (c) soft or (d) hard spring. a solid-state thermal rectifier. ${ }^{38}$ Similarly, by depositing atoms or molecules along the external surface of a CNT following certain distribution, a desired stiffness gradient can be created along the CNT. In our work, to introduce a stiffness gradient, each carbon atom was linked with an underlying spring pointing to the carbon ring center such that the nanotube is connected to a series of springs with linearly graded stiffness $k$ along the nanotube axis direction ( $x$ direction) of the nanotube (cf. Fig. 1b-d). The range of spring stiffness (1 to $11 \mathrm{nN} \mathrm{nm}^{-1}$ ) and stiffness gradient ( 0 to $1 \mathrm{nN} \mathrm{nm}^{-2}$ ) considered in our simulations are comparable with those in previous experiments $^{41}$ and simulations. ${ }^{39,40}$

In the present simulations, five water molecules in a chain configuration were initially placed in the CNT. All simulations were conducted under non-periodic boundary conditions for 50 ps. The system was relaxed for $10 \mathrm{ps}$ at $300 \mathrm{~K}$ with a canonical (NVT) ensemble initially. During the relaxation, the center of mass of the nanotube was kept fixed in all directions, and the center of mass of the water chain was kept fixed along the nanotube axis direction. After that, a microcanonical (NVE) ensemble was adopted for another 40 ps and the confinement on the water chain was released. The integration time step is 1 $\mathrm{fs}$, and the samples of the trajectories were stored every 1 ps.

\section{Results and discussion}

\section{Directional motion of the water chain in the carbon nanotube}

Fig. 2a shows a series of snapshots of the water chain positions at different simulation times, and Video S1 in the ESI $\dagger$ shows the water molecular motion and nanotube fluctuations. In this simulation, the midpoint stiffness $k_{\mathrm{m}}$ is $6 \mathrm{nN} \mathrm{nm}^{-1}$, and the stiffness gradient is $1 \mathrm{nN} \mathrm{nm}{ }^{-2}$. Note that only the right half of the nanotube was plotted and for clarity, the fluctuations of carbon atoms are not shown in Fig. 2a. Our simulation clearly shows that a directional transportation of water molecules can be achieved by the stiffness gradient, and the water molecules move synergistically in a water-chain configuration from the soft region to the stiff region. In the "water-chain", a water molecule is constantly interacting with its adjacent water molecules, in addition to the nanotube wall. To further show that the driving force of the spontaneous directional water transportation was the CNT stiffness gradient, three additional simulations with the same midpoint stiffness $\left(k_{\mathrm{m}}=6 \mathrm{nN} \mathrm{nm}^{-1}\right)$ but smaller gradients $\left(\partial k / \partial x=0,0.4,0.7 \mathrm{nN} \mathrm{nm}^{-2}\right)$ were further carried out (Videos S2 and S3 in the ESI $\dagger$ show the water transportation processes when the stiffness gradient is $0.7 \mathrm{nN}$ $\mathrm{nm}^{-2}$ and 0 , respectively).

Fig. 2b depicts the displacements of the water-molecule chain at different CNT stiffness gradients at every picosecond (details for calculating the displacements are given in the $\mathrm{ESI}_{\dagger} \dagger$ ). It is found that the directional water transportation decreased with decreasing the stiffness gradient, and disappeared when there was no stiffness gradient. The motion of water molecules is not only influenced by the stiffness gradient, but also affected by the absolute value of stiffness of the initial location. We construct a model with water molecules initially located near the beginning of the soft region where the stiffness is $3 \mathrm{nN}$ 

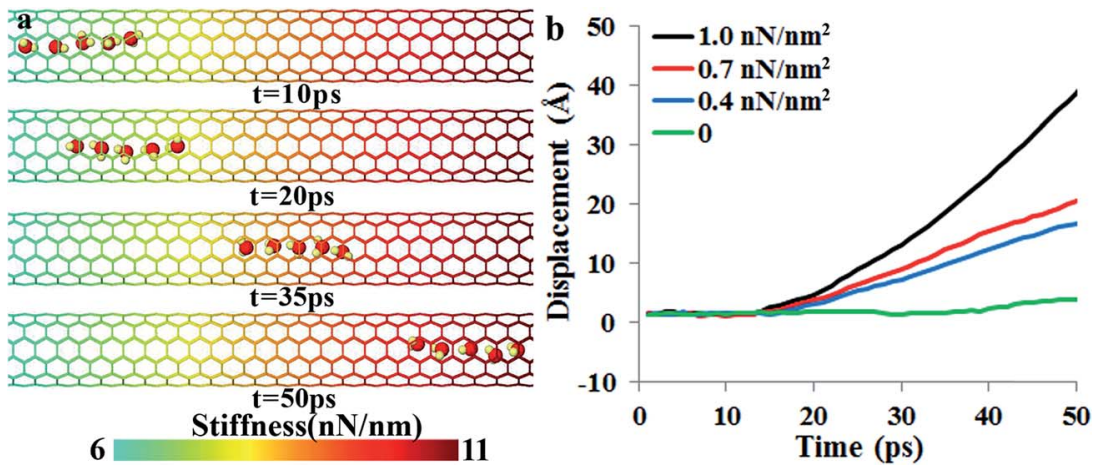

Fig. 2 (a) Snapshots of the positions of a water-molecule chain in a SWCNT at different times. The colors of carbon atoms represent the stiffness of springs that link with the carbon atoms. (b) Displacements of the water-molecule chain as a function of time with different stiffness gradients.

$\mathrm{nm}^{-1}$. The simulation results show that water molecules exhibit a slightly faster movement initially ( $c f$. Fig. S1†), but the spontaneous directional transportation of water molecules and the subsequent speed are independent of the initial location.

\section{Mechanism of directional water transportation induced by the stiffness gradient}

In a mass-loaded CNT, each carbon atom is connected with its three nearest neighbor carbon atoms by covalent bonds, and also to a deposited atom or molecule by a bond (taken as a spring) as shown in Fig. 1. Within the first order approximation, the atomic vibration can be treated as a harmonic one with an effective spring constant. For the out-of-plane mode in the thermal vibration, each carbon atom moves perpendicular to the CNT surface influenced by its three nearest neighbors, and the vibrational amplitude $\Delta R$ is $\left\langle\Delta R^{2}\right\rangle \approx 2 k_{\mathrm{B}} T / K_{\mathrm{e}}$, where $k_{\mathrm{B}}$ is the Boltzmann constant, $T$ the temperature and $K_{\mathrm{e}}$ is the effective spring constant for the carbon atom including the contribution from the $\mathrm{C}-\mathrm{C}$ covalent bonds and the interaction with the deposited mass. Therefore, the amplitude of thermal expansion at a "soft" section is much larger than that at a "stiff" section along the CNT. As a consequence, at the same temperature, with increasing the CNT stiffness, the average distance between the water-chain and CNT wall decreases, leading to a decrease in the potential energy. Therefore, the reduction in potential energy is the underlying physical origin for the directional motion of the water-chain from the "soft" section to the "stiff" section. In our simulations, the system was relaxed with a NVT ensemble initially. After that, a microcanonical NVE ensemble was adopted. In the NVE ensemble, no external thermostat was applied. Therefore, our directional motion is spontaneously achieved by the stiffness gradient without applying external driving forces, which is different from the motion realized by an external thermal gradient ${ }^{13,15}$ and stiffness gradient induced by a thermal gradient. ${ }^{42}$ It is worth noting that although the motion of water molecules inside the CNT is a spontaneous process without resorting to any active driving source, a certain amount of energy is still required to push water molecules entering the CNT, which is the general behaviour for any type of water channel.
To gain further insight into the mechanism, we have calculated the variation of total potential energy for the water-chain and CNT wall as a function of stiffness. Fig. 3a indicates that the total potential energy for the water-chain and CNT wall decreases with increasing the stiffness. Evidently, there is a linear relationship between these quantities. From this linear relationship, we are able to calculate the driving force induced by potential energy reduction:
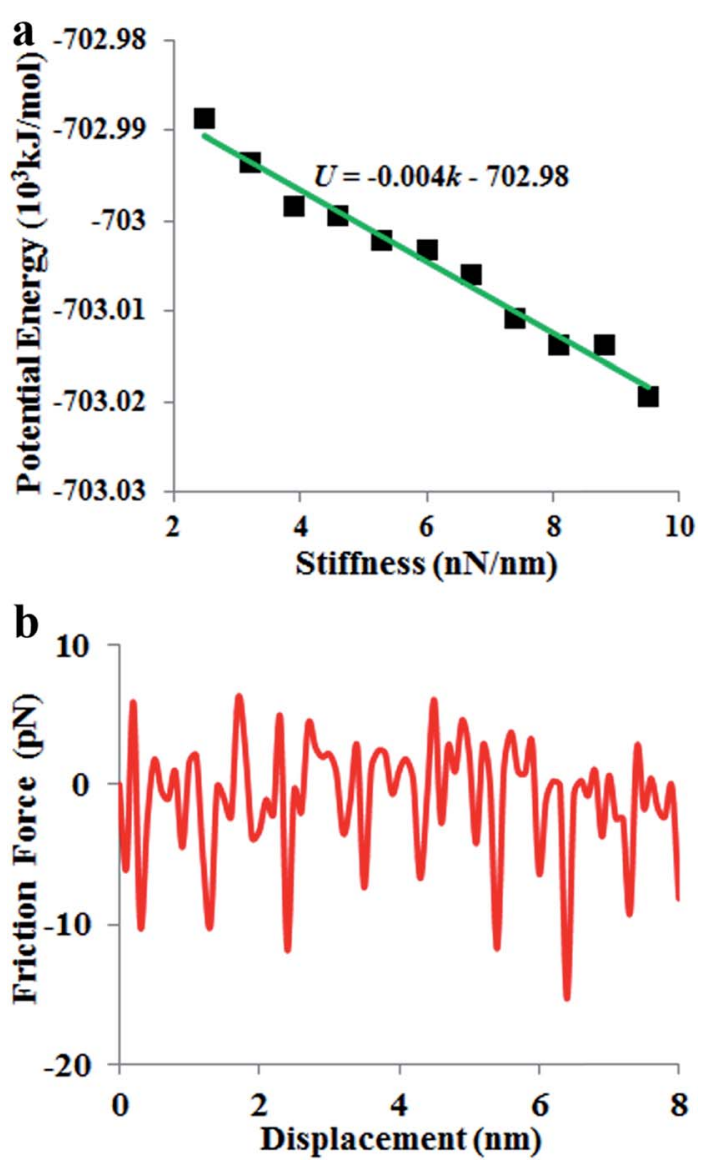

Fig. 3 (a) Variation of potential energy with the nanotube stiffness. (b) Friction force of water molecules moving along the nanotube axis direction. 


$$
F_{\mathrm{d}}(x)=-\frac{\partial U}{\partial x}=-\frac{\partial U}{\partial k} \times \frac{\partial k}{\partial x}
$$

Based on this linear relationship, the average value for the driving force $F_{\mathrm{d}}(x)$ is $\sim 6.6 \mathrm{pN}$.

During the directional motion of the water-chain within the CNT, in addition to the driving force induced by the stiffness gradient, the water-chain also experiences a friction force. ${ }^{43}$ The friction force is shown in Fig. 3b. It is seen that the values of the friction force are in the range from -15 to $5 \mathrm{pN}$. Compared with the aforementioned driving force $(6.6 \mathrm{pN})$ contributed by the stiffness gradient, it is obvious that the driving force is not always larger than friction force during the motion process. The motion of water is similar to the energetically activated stickand-slip motion, which, upon time averaged, resembles a steady-state motion. This phenomenon has also been observed on the motions of large nanoflakes $\left(5 \times 5 \mathrm{~nm}^{2}\right)$ on a substrate, ${ }^{44}$ essentially resembling a particle moving in a periodic potential.

\section{Temperature dependent directional water transportation in carbon nanotubes}

The directional motion of nanoflakes on a substrate with a stiffness gradient has been reported before. ${ }^{39}$ Although the spontaneous motion of water molecules observed in our work is also driven by the reduction in potential energy, there is a fundamental difference in the underlying mechanism. For a graphene nanoflake, there are strong covalent bonds between atoms. Therefore, the nanoflake moves as a whole. In the water chain, however, water molecules are linked by weak hydrogen bonds. Due to thermal vibrations, these hydrogen bonds may break and reform. It is expected that the temperature will have a significant effect on the directional motion of the water-chain.

In the following, we would like to examine the effect of temperature on the directional transportation of water molecules. Fig. 4 shows the positions of water molecules after 50 ps simulation ( $c f$. Fig. 4a), the driving force ( $c f$. Fig. 4b), and the variation of the hydrogen bond ${ }^{45}$ number with time ( $c f$. Fig. 4c) at different temperatures. It is interesting to see that although the water molecules also move in a water-chain manner at $320 \mathrm{~K}$ and $340 \mathrm{~K}$, similar to the case at $300 \mathrm{~K}\left(1.0 \AA \mathrm{ps}^{-1}\right)$, the average velocity is much slower $\left(0.5 \AA \mathrm{ps}^{-1}\right.$ and $\left.0.2 \AA \mathrm{ps}^{-1}\right)$. The underlying mechanism is the suppressed driving force at higher temperature ( $c f$. Fig. $4 \mathrm{~b}$ ). When the temperature increases from $300 \mathrm{~K}$ to $340 \mathrm{~K}$, the driving force decreases from $6.6 \mathrm{pN}$ to 4.0 pN. Moreover, from Fig. 4c, it is clear that at $320 \mathrm{~K}$, the number of hydrogen bonds is predominantly $2-3$, much lower than that at $300 \mathrm{~K}$, signifying the formation of the water-chain only occasionally. With increasing the temperature, the water chain configuration is no longer present in the CNT. For example, the number of hydrogen bonds is only in the range of 1-3 at $340 \mathrm{~K}$, signifying the formation of fragmented smaller chains, but the absence of a complete water chain during the simulation time (cf. Video $\mathrm{S} 4 \dagger)$.

When temperature further increases to $360 \mathrm{~K}(c f$. Video $\mathrm{S} 5 \dagger)$ and $373 \mathrm{~K}$, the driving force is continuously reduced $(2.8 \mathrm{pN}$ and $1.8 \mathrm{pN}$ in Fig. 4b). Correspondingly, Fig. 4a indicates that the directional water transportation is suppressed at $360 \mathrm{~K}$ and 373 K. Fig. 4c indicates that the number of hydrogen bonds is in the range of $0-2$ at $360 \mathrm{~K}$ and $373 \mathrm{~K}$, signifying further fragmentation of water chains and the absence of any complete water chain. The fragmentation of water chains, which is caused by the promoted thermal fluctuations, is also visible in Fig. 4a. A direct consequence of chain fragmentation is the reduction in the motion velocity of the water molecules, as shown in Video S4 (340 K) and S5 (360 K) in the ESI. $\dagger$ Our results clearly suggest that for a single water molecule, its "effective" length along the potential gradient field is limited, and thus the "effective" driving force is small. On the other hand, by forming the waterchain configuration, the "effective" length is remarkably increased, and therefore the water molecules can move spontaneously and collectively.
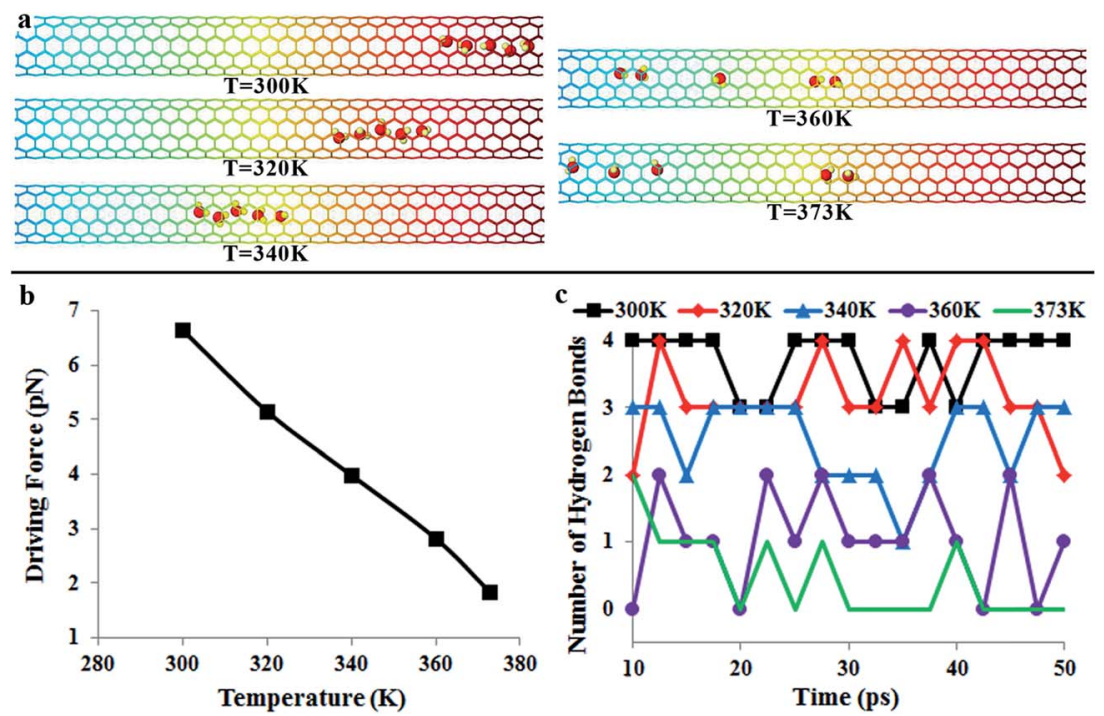

Fig. 4 (a) Snapshots of the positions of water molecules after 50 ps simulation, (b) driving force to transport water molecules, and (c) variation of the number of hydrogen bonds with time at different temperatures. 

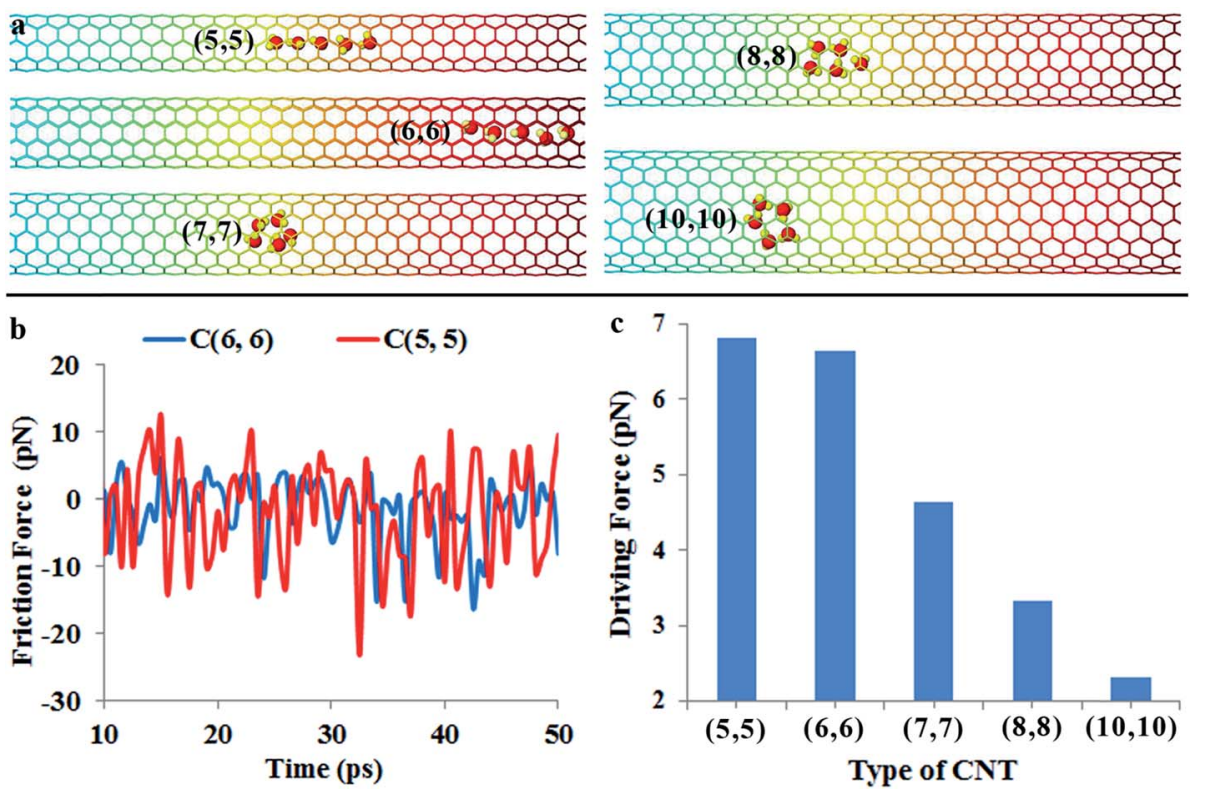

Fig. 5 (a) Snapshots of the positions of water molecules after 50 ps simulation in $(5,5),(6,6),(7,7),(8,8)$ and $(10,10)$ CNTs. (b) Friction force of water molecules moving along the nanotube axis direction in $(5,5)$ and $(6,6) \mathrm{CNTs}$. (c) Driving force to transport water molecules in $(5,5),(6,6)$, $(7,7),(8,8)$ and $(10,10)$ CNTs.

\section{Diameter dependent directional water transportation in carbon nanotubes}

Nowadays, the chirality and diameter of single-walled carbon nanotubes can be controlled accurately during growth. ${ }^{28,29}$ This enables one to study water transportation in different sized CNTs. Here, we examine the effect of CNT diameter on the water transport behavior using MD simulations. Fig. 5a shows the positions of water molecules in $(5,5),(6,6),(7,7),(8,8)$ and $(10$, 10) CNTs (their corresponding diameters are $6.68 \AA$, $8.02 \AA, 9.36$ $\AA, 10.70 \AA$ and $13.37 \AA$, respectively) after 50 ps simulation. For the $(5,5)$ and $(6,6)$ CNTs, it is found that the water molecules are still able to keep the chain configuration. The motion of water molecules in the $(5,5) \mathrm{CNT}$ ( $c f$. Video S6 in the ESI $\dagger$ ) is obviously slower than that in the $(6,6)$ CNT even if the driving force increases slightly from $6.6 \mathrm{pN}$ for the $(6,6) \mathrm{CNT}$ to $6.8 \mathrm{pN}$ for the $(5,5)$ CNT ( $c f$. Fig. 5c). When a water molecule moves within a CNT, it essentially resembles a particle moving in a periodic potential field, and the repeating energy barrier is the origin of the friction force. The radius of the $(5,5) \mathrm{CNT}$ is about $3.34 \AA$, which is comparable to the equilibrium distance $(3.2 \AA)$ between the water molecule and graphene sheet governed by van der Waals interaction. ${ }^{46}$ Therefore, there is a strong confinement of the $(5,5) \mathrm{CNT}$ on the water molecules. As a result, the potential energy field of the CNT wall can strongly distort the water-molecule chain, giving rise to a slower motion velocity. This is consistent with the larger friction force in the $(5$, 5) CNT ( $c f$. Fig. 5b).

On the other hand, the water transportation is also suppressed in larger carbon nanotubes even if the friction force is lower than that in the $(6,6)$ nanotube $(c f$. Fig. S2, Video S7 and S8 in the ESI $\dagger$ ). The reason is that the driving force resulted from the gradient of potential energy decreases remarkably in larger nanotubes ( $c f$. Fig. 5c). Moreover, when the diameter of the nanotube increases, the confinement of the CNT on water molecules becomes weaker, leading to increased movement space and flexibility for water molecules. In that case, water molecules are able to form a ring structure instead of a chain configuration. Moreover, the weak confinement permits more degrees of freedom, including the rotation degree of freedom and lateral space degree of water molecules to explore. The enhanced transversal and rotational degrees of freedom significantly reduce the spontaneous motion of water molecules.

\section{Conclusions}

In summary, the transport behavior of water molecules in a single-walled carbon nanotube with a stiffness gradient was studied by molecular dynamics simulations. A spontaneous directional transportation of water molecules from a "soft" region to a "stiff" region is achieved. The water transport rate is optimal in the $(6,6)$ CNT. The directional transportation decreased with decreasing the stiffness gradient, and disappeared when there was no stiffness gradient. The underlying mechanism was attributed to the total potential energy difference induced by the stiffness gradient. Furthermore, a theoretical calculation including the forces that water molecules experienced was carried out. Related analyses on driving force and friction force were made. Other factors such as temperature and diameter were also investigated. Our study has clearly shown that a CNT with a stiffness gradient is able to realize the spontaneous directional motion of water molecules, without resorting to any active driving force, such as electric field, pressure or temperature difference. This new way of controlling 
water molecules at the nanoscale may have potential applications in biology, nanofluidic devices, water purification, drug delivery, seawater desalination, nanofiltration, etc.

\section{Conflicts of interest}

There are no conflicts to declare.

\section{Acknowledgements}

The authors gratefully acknowledge the financial support from the Agency for Science, Technology and Research (A*STAR), Singapore and the use of computing resources at the A*STAR Computational Resource Centre, and National Supercomputing Centre, Singapore. The authors are supported in part by a grant from the Science and Engineering Research Council (152-7000017).

\section{References}

1 K. Murata, K. Mitsuoka, T. Hirai, T. Walz, P. Agrek, J. B. Heymann, A. Engel and Y. Fujiyoshi, Nature, 2000, 407, 599-605.

2 G. Hummer, J. C. Rasaiah and J. P. Noworyta, Nature, 2001, 414, 188-190.

3 K. Besteman, J. O. Lee, F. G. M. Wiertz, H. A. Heering and C. Dekker, Nano Lett., 2003, 3, 727-730.

4 M. A. Shannon, P. W. Bohn, M. Elimelech, J. G. Georgiadis, B. J. Marinas and A. M. Mayes, Nature, 2008, 452, 301-310.

5 J. Shi, A. R. Votruba, O. C. Farokhzad and R. Langer, Nano Lett., 2010, 10, 3223-3230.

6 M. Elimelech and W. A. Phillip, Science, 2011, 33, 712-717.

7 R. R. Nair, H. A. Wu, P. N. Jayaram, I. V. Grigorieva and A. K. Geim, Science, 2012, 335(6067), 442-444.

8 S. C. O'Hern, D. Jang, S. Bose, J. C. Idrobo, Y. Song, T. Laoui, J. Kong and R. Karnik, Nano Lett., 2015, 15, 3254-3260.

9 Y. Zhu, F. Wang, J. Bai, X. C. Zeng and H. Wu, ACS Nano, 2015, 9(12), 12197-12204.

10 M. Ma, G. Tocci, A. Michaelides and G. Aeppli, Nat. Mater., 2016, 15, 66-71.

11 J. Li, X. Gong, H. Lu, D. Li, H. Fang and R. Zhou, Proc. Natl. Acad. Sci. U. S. A., 2007, 104, 3687-3692.

12 X. Gong, J. Li, H. Lu, R. Wan, J. Li, J. Hu and H. Fang, Nat. Nanotechnol., 2007, 2, 709-712.

13 A. Barreiro, R. Rurali, E. R. Hernández, J. Moser, T. Pichler, L. Forro and A. Bachtold, Science, 2008, 320, 775-778.

14 G. Zuo, R. Shen, S. Ma and W. Guo, ACS Nano, 2010, 4, 205210.

15 E. Oyarzua, J. H. Walther, C. M. Megaridis, P. Koumoutsakos and H. A. Zambrano, ACS Nano, 2017, 11(10), 9997-10002.

16 X. Gong, J. Li, H. Zhang, R. Wan, H. Lu, S. Wang and H. Fang, Phys. Rev. Lett., 2008, 101, 257801.

17 X. Li, Y. Shi, Y. Yang, H. Du, R. Zhou and Y. Zhao, J. Chem. Phys., 2012, 136, $05 \mathrm{~B} 602$.

18 J. Zou, B. Ji, X.-Q. Feng and H. Gao, Nano Lett., 2006, 6(3), 430-434.
19 Z. Cui, D. Li and B. Ji, Int. J. Appl. Mech. Eng., 2015, 07(06), 1550084.

20 X. Ma, S. Cambré, W. Wenseleers, S. K. Doorn and H. Htoon, Phys. Rev. Lett., 2017, 118(2), 027402.

21 K. V. Agrawal, S. Shimizu, L. W. Drahushuk, D. Kilcoyne and M. S. Strano, Nat. Nanotechnol., 2017, 12(3), 267-273.

22 M. Nishi, T. Ohkubo, K. Urita, I. Moriguchi and Y. Kuroda, Langmuir, 2016, 32(4), 1058-1064.

23 K. Nomura, T. Kaneko, J. Bai, J. S. Francisco, K. Yasuoka and X. C. Zeng, Proc. Natl. Acad. Sci. U. S. A., 2017, 114(16), 40664071.

24 S. D. Bernardina, E. Paineau, J. B. Brubach, P. Judeinstein, S. Rouzière, P. Launois and P. Roy, J. Am. Chem. Soc., 2016, 138(33), 10437-10443.

25 M. Ma, F. Grey, L. Shen, M. Urbakh, S. Wu, J. Z. Liu, Y. Liu and Q. Zheng, Nat. Nanotechnol., 2015, 10, 692-695.

26 K. Goh and Y. Chen, Nano Today, 2017, 14, 13-15.

27 R. H. Tunuguntla, R. Y. Henley, Y. C. Yao, T. A. Pham, M. Wanunu and A. Noy, Science, 2017, 357(6353), 792-796.

28 S. Zhang, L. Kang, X. Wang, L. Tong, L. Yang, Z. Wang, K. Qi, S. Deng, Q. Li, X. Bai, F. Ding and J. Zhang, Nature, 2017, 543(7644), 234-238.

29 F. Yang, X. Wang, D. Zhang, J. Yang, D. Luo, Z. Xu, J. Wei, J.-Q. Wang, Z. Xu, F. Peng, X. Li, R. Li, Y. Li, M. Li, X. Bai, F. Ding and Y. Li, Nature, 2014, 510(7506), 522-524.

30 D. Dundas, E. J. McEniry and T. N. Todorov, Nat. Nanotechnol., 2009, 4, 99-102.

31 O. E. Shklyaev, E. Mockensturm and V. H. Crespi, Phys. Rev. Lett., 2013, 110, 156803.

32 M. G. L. van den Heuvel and C. Dekker, Science, 2007, 317, 333-336.

33 A. S. Barnard, Nature, 2015, 519(7541), 37-38.

34 S. Plimpton, J. Comput. Phys., 1995, 117, 1-19.

35 S. J. Stuart, A. B. Tutein and J. A. Harrison, J. Chem. Phys., 2000, 112, 6472-6486.

36 W. L. Jorgensen, J. Chandrasekhar, J. D. Madura, R. W. Impey and M. L. Klein, J. Chem. Phys., 1983, 79, 926935.

37 J. E. Lennard-Jones, Proc. R. Soc. London, 1924, 106(738), 463-477.

38 C. W. Chang, D. Okawa, A. Majumdar and A. Zettl, Science, 2006, 314(5802), 1121-1124.

39 T. Chang, H. Zhang, Z. Guo, X. Guo and H. Gao, Phys. Rev. Lett., 2015, 114, 015504.

40 M. Becton and X. Wang, RSC Adv., 2016, 6(56), 51205-51210. 41 R. Li, H. Ye, W. Zhang, G. Ma and Y. Su, Sci. Rep., 2015, 5, 15828.

42 N. R. Raravikar, P. Keblinski, A. M. Rao, M. S. Dresselhaus, L. S. Schadler and P. M. Ajayan, Phys. Rev. B, 2002, 66(23), 235424.

43 P. Liu and Y. W. Zhang, Carbon, 2011, 49, 3687-3697.

44 Y. Cheng, G. Zhang, Y. Zhang, T. Chang, Q. X. Pei, Y. Cai and Y. W. Zhang, Nanoscale, 2018, 10, 1660-1666.

45 H. Xu and B. J. Berne, J. Phys. Chem. B, 2001, 105, 1192911932.

46 T. Werder, J. H. Walther, R. L. Jaffe, T. Halicioglu and P. Koumoutsakos, J. Phys. Chem. B, 2003, 107, 1345-1352. 